\title{
Variabilidad y calidad de la práctica clínica de enfermería en pacientes con Diabetes mellitus, hospitalizados
}

Perla Simons Morales ${ }^{1}$

\section{RESUMEN}

El estudio analiza la variabilidad y la calidad de la práctica clínica de Enfermería en pacientes que padecen Diabetes mellitus, hospitalizados en las salas de Medicina Interna del Hospital Escuela Universitario. Tegucigalpa, Honduras. C.A. agosto a noviembre, 2015. Utilizando un método mixto, la muestra recursos humanos de enfermería, licenciadas y auxiliares y pacientes hospitalizados en el enfoque cuantitativo se encuestaron 54 auxiliares de enfermería y 6 licenciadas de enfermería en el enfoque cualitativo 4 auxiliares, 3 licenciadas en enfermería y 10 pacientes con diagnóstico de diabetes mellitus. Entre los resultados podemos la variabilidad en la práctica de enfermería a los pacientes diabéticos. En estas variaciones de la atención inciden factores que tiene que ver con características propias del personal de enfermería que participo en el estudio (años de experiencia laboral y nivel de conocimientos sobre el problema de salud que adolecen las personas que atienden).

El 100\% del personal de enfermería refirieron nunca haber recibido capacitación sobre cuidados a pacientes con Diabetes mellitus. En las salas no existen protocolos, guías clínicas o normas de enfermería que estandaricen el cuidado en pacientes con diabetes mellitus.

Respecto a la calidad de la atención de enfermería desde la percepción de los pacientes fue satisfactoria.

Palabras claves: variabilidad, calidad, práctica clínica de enfermería, Diabetes mellitus

1 Beneficiaria de una beca básica de la DICYP. Profesora de la Carrera de Enfermería, Facultad de Ciencias Médicas, UNAH :simonsperla@yahoo.es 


\section{ABSTRACT}

The study analyzes the variability and quality of the practice of clinical nursing with patients that suffer Diabetes mellitus, hospitalized in the Internal Medicine in the University's School Hospital from the period August to November, 2015. Using a mixed method, the sample included nursing workforce, graduates, assistants, and hospitalized patients. In the quantitative research, 54 nursing assistants and 6 nursing graduates were surveyed; in the qualitative approach 4 assistants, 3 nursing graduates and 10 patients diagnosed with Diabetes mellitus. Among the results we found variability in the practice of nursing diabetic patients. These variations of care are affected by factors that have to do with each subject's personal characteristics (years of labor experience and level of knowledge about the health problem that each patient suffers.) $100 \%$ of the nursing personal said they never received any training about how to take care of the patients suffering diabetes mellitus. There were no protocols available in the wards, nor clinical guidelines and nursing norms that standardized the care of patients with Diabetes mellitus.

Regarding the quality of attention provided by the nurses, the patients were satisfied.

Key words: variability, quality, clinic practice of nursing, Diabetes mellitus. 


\section{INTRODUCCIÓN}

La gestión clínica, nos lleva a la mejora de los cuidados y se concibe como una herramienta de innovación necesaria de los servicios de cara a mejorar la eficacia, efectividad y eficiencia en la prestación para atender a las personas que padecen una enfermedad, partiendo de la capacidad que tienen los profesionales de organizarse con autonomía y responsabilidad.

La vigilancia de la calidad con que se brinda la atención en los servicios de salud es uno de los procesos de mayor importancia para asegurar el cumplimiento de los objetivos en pro de la salud del paciente así como también para reducir complicaciones y muertes.

Para desarrollar la gestión clínica debe existir un modelo que este en concordancia con la evidencia científica. Este marco de trabajo supone que los objetivos de eficiencia deben estar alrededor del desarrollo profesional y ético de la persona. A nivel organizativo la gestión clínica implica dotar a una unidad asistencial de una estructura pequeña de gestión, con autoridad e instrumentos para planificar y gestionar sus actividades.

Es importante reconocer que enfermería debe gestionar la calidad dentro de su desempeño.

Calidad: Es la capacidad de satisfacer o superar las necesidades o expectativas de aquellos que están demandando o prestando estos servicios, en forma congruente con un estándar básico de referencia. Por ello la calidad puede ser vista como un estilo o filosofía de vida en un mundo que está cambiando y evolucionando cada día. (Cañarejo Andrango Andrea Estefanía, 2013).

"La calidad científico-técnica de la actividad clínica obliga a tener para su optimización una base sólida constituida por las mejores evidencias científicas disponibles". (L. Quecedo, 2001)

La calidad depende de un juicio que realiza el usuario sobre un producto o servicio obtenido según las características de su uso y la urgencia de poseerlo. Cuando el producto 0 el servicio entran en contacto con el usuario, la calidad se torna en una variable valorativa propia de la interrelación entre los dos. De esta manera, el producto o servicio puede adquirir diferentes valores en la medida en que responda o 
no a la búsqueda del usuario o cliente. (Heredia, 2015)

A fin de desarrollar conocimientos que favorezcan la puesta en marcha de estrategias para mejorar la gestión de los servicios de enfermería en el manejo de pacientes con diagnóstico de Diabetes mellitus en Honduras, se tomó a bien evidenciar la calidad de atención que brindan estos profesionales de la salud a pacientes con el diagnostico de esta enfermedad.

La calidad para brindar los cuidados al paciente que padece Diabetes mellitus en Honduras, se constituye en un reto para los profesionales de enfermería, debido a los factores que inciden alrededor de la atención que se brindan a este tipo de pacientes, entre ellos: las características propias del paciente diabético, su condición en la evolución de la enfermedad, se debe tomar en cuenta que ellos son proclives a padecer otras enfermedades que se desencadenan con el tiempo, derivadas de la cronicidad de la enfermedad como las cardiopatías, accidentes cerebrovasculares, neuropatías etc., esto implica que se requiere de la 'preparación profesional y un alto grado de desarrollo de competencias procedimentales.

Considerando el alto costo socioeconómico que esta enfermedad provoca en el sistema de salud hondureño y dado a que enfermería brinda atención directa a estos pacientes, es relevante valorar desde las dimensiones del cuidado, cual es el papel de enfermería en la mejora de la condición de salud de ellos.

En cuanto a las dimensiones de la calidad del servicio y a su medición se identifican dimensiones que representan los criterios que utilizan los consumidores en el proceso de evaluación de la calidad en cualquier tipo de servicio: elementos tangibles (apariencia física de las instalaciones, equipos, personal, etc.), fiabilidad (habilidad para ejecutar el servicio prometido de forma fiable y cuidadosa desde la primera vez), capacidad de respuesta (disposición de ayudar y servir rápidamente a los clientes), seguridad (habilidades y conocimientos de los empleados para inspirar credibilidad y confianza) y empatía (atención individualizada que ofrecen las empresas a sus clientes). (Mauricio Losada Otalora, 2007)

La calidad en la práctica clínica de salud tiene dimensiones cuyo propósito es apoyar la monitoria y seguimiento de los procesos que se desarrollan alrededor de los cuidados a los pacientes. Dentro de las dimensiones están:

Competencia profesional: que es la capacidad del personal de enfermería, para utilizar los más avanzados conocimientos para realizar las tareas en forma uniforme 
y precisa (desempeño real), tecnología y recursos disponibles, para resolver los problemas de salud de los pacientes y producir su máxima satisfacción y seguridad de la atención.

Se relaciona con la ejecución de las pautas y normas para el ejercicio de la profesión y la realización en términos de habilidad, precisión, confiabilidad y uniformidad. Comprende las técnicas relacionadas con el diagnóstico y el tratamiento así como la capacidad de suministrar asesoramiento efectivo en salud y establecer una buena relación con el paciente.

La experiencia que precisa el personal de apoyo depende de cada descripción del puesto, aunque las técnicas de relación interpersonal con el paciente y las comunidades son de particular importancia.

La falta de competencia profesional puede variar de desviaciones menores de los procedimientos normales a errores de importancia que disminuyen la eficacia 0 ponen en peligro la seguridad del paciente.

Se espera que el personal de enfermería desarrolle actuaciones (preventivas, diagnósticas terapéuticas y de cuidados) a través de las cuales se establezcan los objetivos de control y plan terapéutico, educación diabetológica, prevención y tratamiento de las complicaciones y rehabilitación, cuando las complicaciones ya están presentes.

La práctica clínica que enfermería realiza debe evidenciar ser de calidad y estar fundamentada en evidencias científicas. Esta condición implica poseer conocimiento técnico científico y el desarrollo de competencias específicas para brindar servicios efectivos, oportunos, coherentes a las necesidades de salud de los pacientes y eficientes para la institución de salud.

Se espera que enfermería realice una atención fiable es decir brindar un servicio de manera confiable, segura y cuidadosa. La seguridad del paciente puede ser evaluada a través de 10 aspectos, estos aspectos son la identificación del paciente, comunicación clara, manejo de medicamentos, cirugía o procedimiento correcto, evitar caída del paciente, uso de protocolos y guías, evitar infecciones nosocomiales, clima de seguridad, factores humanos, hacer partícipe al paciente en la toma de decisiones. (Cañarejo Andrango Andrea Estefanía, 2013).

La práctica de enfermería de calidad también observa la adopción de un modelo para 
aplicar el proceso de atención de enfermería como herramienta científica de la profesión, la aplicación del cuidado con base en un modelo teórico facilita el uso de un lenguaje común o estandarizado para la práctica. (Juan Reyes Luna, 2007). Al mismo tiempo se necesita identificar indicadores que permitan medir la calidad del cuidado de enfermería.

Muir Gray propone que la toma de decisiones sobre la atención sanitaria que afecte a grupos de pacientes o poblaciones, debe realizarse combinando tres factores: evidencias, valores y recursos. (Jesus Lopez Alcalde, 2008).

La Diabetes mellitus es una enfermedad de gran impacto social, está en aumento en los países en desarrollo debido a los estilos de vida y envejecimiento progresivo de la población, es considerado un problema de salud pública por las complicaciones agudas y crónicas que producen disminución en la calidad de vida como lo son la limitación funcional y en algunos casos se desenlaza hasta la muerte.

La Diabetes mellitus aparece cuando el páncreas no produce insulina suficiente 0 cuando el organismo no utiliza eficazmente la insulina que produce. La insulina es una hormona que regula el azúcar en la sangre.

El efecto de la diabetes no controlada es la hiperglucemia (aumento del azúcar en la sangre), que con el tiempo daña gravemente muchos órganos y sistemas, especialmente los nervios y los vasos sanguíneos. (Salud, 2014, pág. 01).

En Honduras se desconocen estudios que analicen la calidad de los cuidados de enfermería en personas que padecen Diabetes mellitus y de la existencia de variaciones en los cuidados que realizan a pacientes con Diabetes mellitus.

Las variaciones o variabilidad, se definen como aquellas diferencias en el proceso asistencial y/o en el resultado de la atención de un problema clínico concreto, entre diversos proveedores o uno mismo, una vez controlados los factores demográficos, socioculturales y de estado de salud" (Toro, 2005). Estas diferencias a las que nos referimos están más en relación con los distintos estilos de práctica profesional.

Según Bernar la hipótesis de la incertidumbre predice que la variabilidad en la práctica clínica será escasa cuando exista acuerdo entre los clínicos sobre el valor de un procedimiento; en presencia de incertidumbre cabe la posibilidad de que cada profesional adopte una decisión diferente. (Hueso, 2015) En estos casos es cuando la oferta de servicios (volumen de camas, número de médicos, recursos materiales 
disponibles, etc.).

La información que se pueda obtener, sobre las prácticas del cuidado que enfermería realiza a pacientes con Diabetes mellitus en el Hospital Escuela Universitario, apoyaría a brindar evidencia para construir parámetros de medición en la eficacia, eficiencia y efectividad de los cuidados brindados a sabiendas que estos inciden directamente en la recuperación de la salud de los pacientes mencionados.

Es imprescindible conocer el modelo de atención que desarrolla el personal de enfermería en pacientes con Diabetes mellitus, los criterios científicos y técnicos que fundamentan la toma de decisiones para su actuación en el manejo terapéutico, tener conocimiento si la práctica clínica que realiza está sustentada en Guías, Protocolos, normas de atención propias de la profesión, las mismas que permitan llevar un estándar de calidad en la práctica y por ende disminuir incertidumbre y variabilidad de la misma.

Los diferentes estilos profesionales pueden llevar no sólo a un diferente e injustificado consumo de recursos, sino también a diferencias en los resultados en salud.

La existencia de indicadores institucionales que evidencien el quehacer de estos profesionales y fortalezcan la monitoria y valuación del desarrollo del proceso de atención dirigido a pacientes diabéticos hospitalizados.

Cabe resaltar la importancia de conocer el grado de efectividad con el cual enfermería resuelve los problemas de salud de pacientes con Diabetes mellitus hospitalizados, la pertinencia en la toma de decisiones en las competencias profesionales e identificar la existencia de diferencias injustificadas en las actuaciones clínicas (variabilidad) y si los factores anteriores cumplen con las características básicas para lograr la satisfacción de los pacientes y familiares.

\section{METODOLOGÍA}

Se realizó estudio mixto (CUAN - cual), la información cuantitativa y cualitativa fue obtenida en las seis salas de Medicina Interna A, B y $C$ de hombres y mujeres en el Hospital Escuela Universitario.

Método cuantitativo: Diseño no experimental. Descriptivo, transversal 
Muestra cuantitativa: No probabilística a conveniencia, de un total de 85 personas que laboran en el departamento de Enfermería en las 6 salas del estudio se tomaron: personal auxiliar de enfermería (54) y profesional de enfermería (6) en un total de (60). Se abordó todo el personal de enfermería que se encontraba laborando en las salas de Medicina Interna en el mes de agosto de 2015 y que brindaban atención a pacientes hospitalizados con diagnóstico de Diabetes mellitus. No se incluyó personal de Enfermería de vacaciones, licencias o incapacidad médica.

\section{Método cualitativo: Narrativa}

Muestra cualitativa: Fueron (10) Pacientes con Diabetes mellitus hospitalizados en las salas de Medicina Interna del HEU. (Medicina de hombres y Mujeres). Se obtuvo la información a través de grabaciones en audio, se identificaron las percepciones sobre la calidad de los cuidados que reciben de enfermería. Se excluyeron del estudio aquellos pacientes que por sus condiciones cognitivas no pudieran ser entrevistados. De los participantes entrevistados el $60 \%(6)$ fueron del sexo femenino y el $40 \%(4)$ del sexo masculino. El 70\% (7), en cuanto a número de hospitalizaciones de los participantes estaban hospitalizados por primera vez, un $20 \%$ (2), por segunda vez y un $10 \%$ (1) se habían hospitalizado más de dos veces.

Los factores que inciden en la variabilidad de la práctica de enfermería fueron abordados a profundidad con (2) licenciadas de Enfermería y (4) auxiliares de enfermería que laboran en las salas de Medicina Interna del HEU. (Medicina de hombres y Mujeres) y que realizan su práctica clínica en pacientes con diagnóstico de diabetes mellitus.

Previa ejecución del estudio se obtuvo autorización de las autoridades de Enfermería del Hospital Escuela Universitario, se programó y coordino la realización de la prueba piloto y recolección de información; en el enfoque cuantitativo la encuesta y el desarrollo de entrevistas en el enfoque cualitativo. La recolección de información se realiza previo consentimiento informado de los participantes al igual que las grabaciones de las entrevistas. Se hizo énfasis con ellos que se guardaría confidencialidad de los informantes y la información.

Los datos cuantitativos se manejaron con el software Epi. Info. 7 y la información cualitativa fue organizada en categorías, codificada con el software Atlas Ti para su posterior análisis. 


\section{Resultados de la investigación}

El 98\% (54) del personal encuestado era del sexo femenino y el $2 \%$ (6) del sexo masculino. Las edades estaban comprendidas el 36\% (22) en rangos entre 34 a 43 años, un $27 \%$ (16) en edades de 44 a 53 años, un $27 \%$ (16) en edades de 22 a

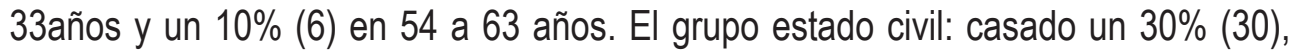
solteros $23 \%$ (14), unión libre $18 \%$ (11) y no consignaron estatus un $9 \%$ (5).

El 37\%(22) eran empleadas con antigüedad laboral de 5 a 15 años, un (18) $30 \%$, antigüedad de 1 a 4 años el (9)15\% han laborado más de 21 años, menos de un año un (6) $10 \%$ y un $8 \%$ han laborado de 16 a 20años. El $88 \%$ (47) tiene una condición laboral permanente y un $12 \%$ (13) están por contrato. De las salas estudiadas un $80 \%$ cuentan con 1 licenciada de Enfermería por turno y un (2) $3 \%$ de las salas se encontraban sin licenciada en Enfermería.

\section{Disponibilidad del recurso humano y ocupación de camas en las salas de Medi- cina Interna HEU.}

Según el 95\%(57) del personal entrevistado el día que se desarrolló la encuesta se disponía en el turno "A "de (1) licenciada de enfermería y más de (2) auxiliares de enfermería, el $80 \%$ (48) de los encuestados admitieron que la sala en la que laboran se encuentran con una ocupación de camas del 100\% (34). La ocupación de camas de las salas estudiadas estuvo en mayor frecuencia entre 32,33 y 31 respectivamente.

El 100\%(60) del personal consideran que la disponibilidad de recursos humanos de enfermería para dar respuesta a la demanda de pacientes es insuficiente para brindar cuidados de calidad.

Fundamentación de las intervenciones de enfermería: Las intervenciones enfermeras en pacientes diabéticos se fundamentan principalmente el $43 \%(26)$ en un modelo médico, así como también el diagnostico medico es el primer criterio para brindar los cuidados. Según la valoración realizada con cada paciente se establecen los diagnósticos enfermeros (diagnostico real, diagnostico de riesgo y diagnóstico de bienestar) que van a diferir de unos pacientes a otros. A partir de ellos se inicia una serie de criterios de resultados, intervenciones y de actividades enfermeras. 
Tabla 1. Valoración de Enfermería al paciente interno en sala con diagnóstico de Diabetes mellitus

\begin{tabular}{|l|l|l|l|}
\hline Descripción & Características & $\mathbf{N}^{\mathbf{a}}$ & $\%$ \\
\hline Valoración & Percepción del paciente sobre su estado de salud & 30 & 50 \\
\hline & Autocuidado que realiza el paciente & 29 & 48 \\
\hline & $\begin{array}{l}\text { Percepción cognición del paciente } \\
\text { Estado de orientación }\end{array}$ & 34 & 57 \\
\hline & Alteración de la memoria & 13 & 22 \\
\hline & Déficit de la comunicación & 7 & 12 \\
\hline
\end{tabular}

Fuente: Encuestas estudio de Variabilidad y calidad de la práctica de enfermería en pacientes con Diabetes mellitus en salas de Medicina Interna, HEU.

\section{Grafica1. Valoración estado nutricional del paciente con DM.}

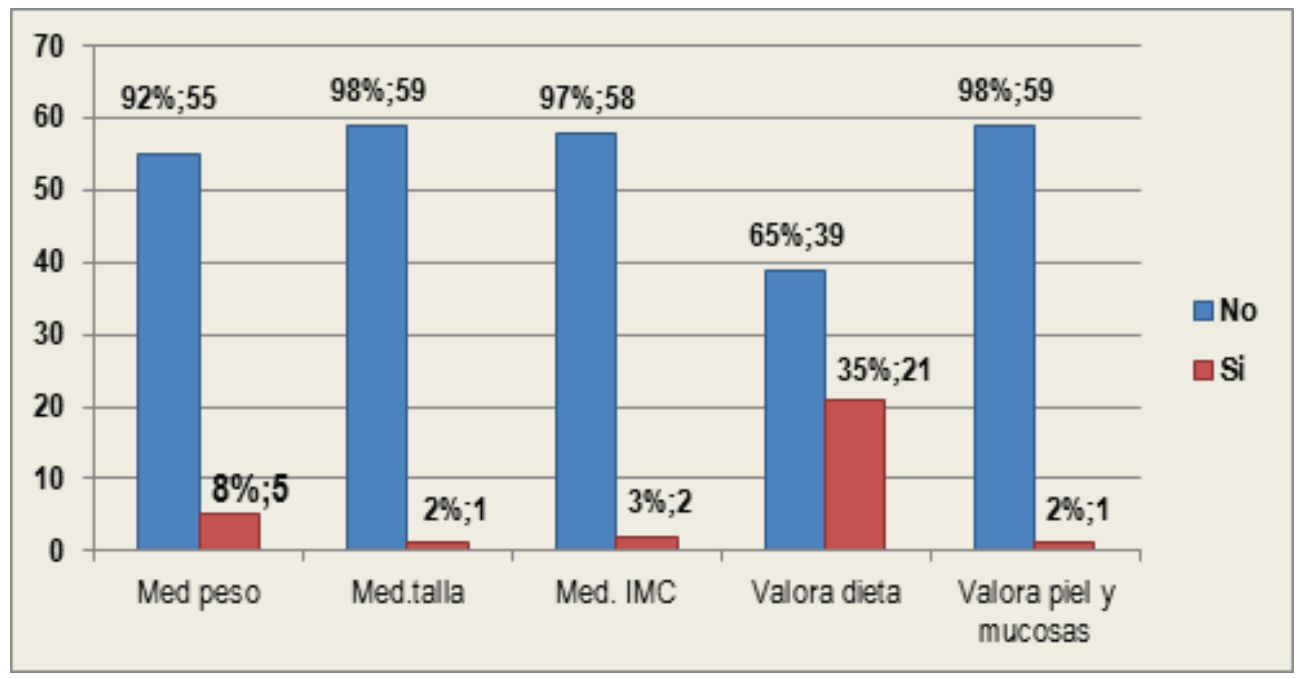

Fuente: Encuestas estudio de Variabilidad y calidad de la práctica de enfermería en pacientes con Diabetes mellitus en salas de Medicina Interna, HEU. 
Grafica 2. Intervenciones de enfermería a pacientes con signos de hiperglicemia

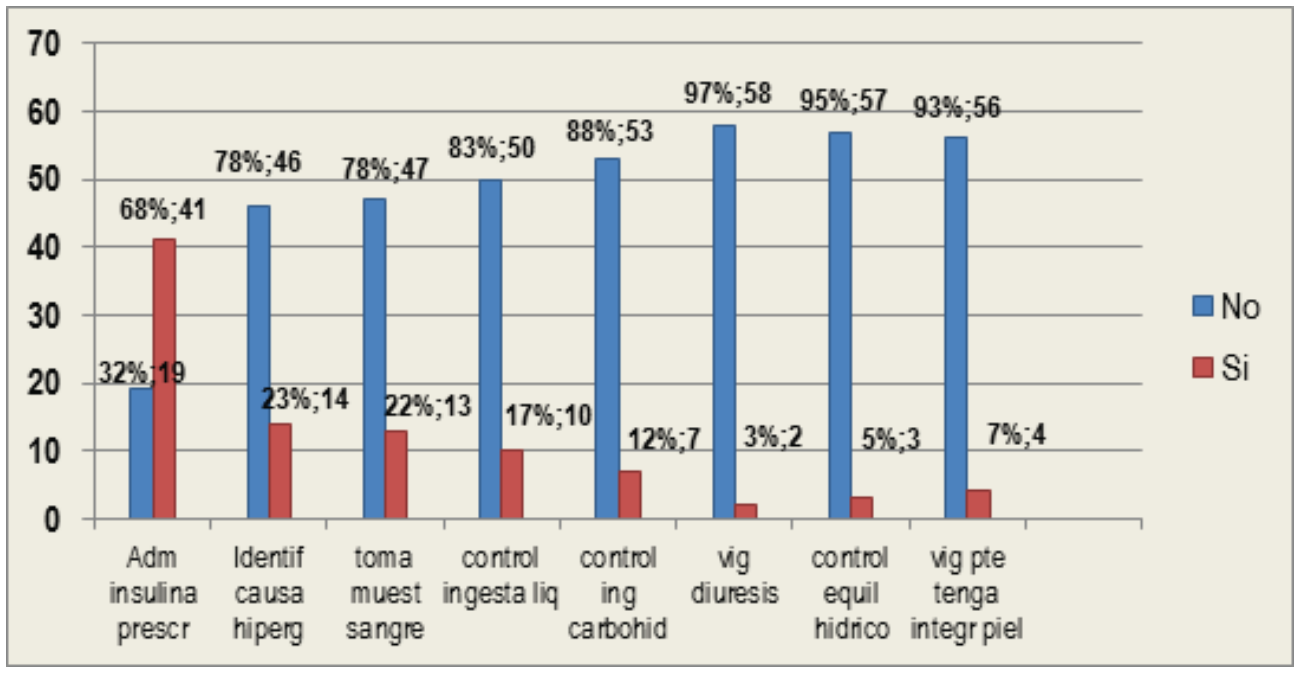

Fuente: Encuestas estudio de Variabilidad y calidad de la práctica de enfermería en pacientes con Diabetes mellitus en salas de Medicina Interna, HEU.

\section{Grafica 3. Intervenciones de enfermería a pacientes con signos de hipoglicemia}

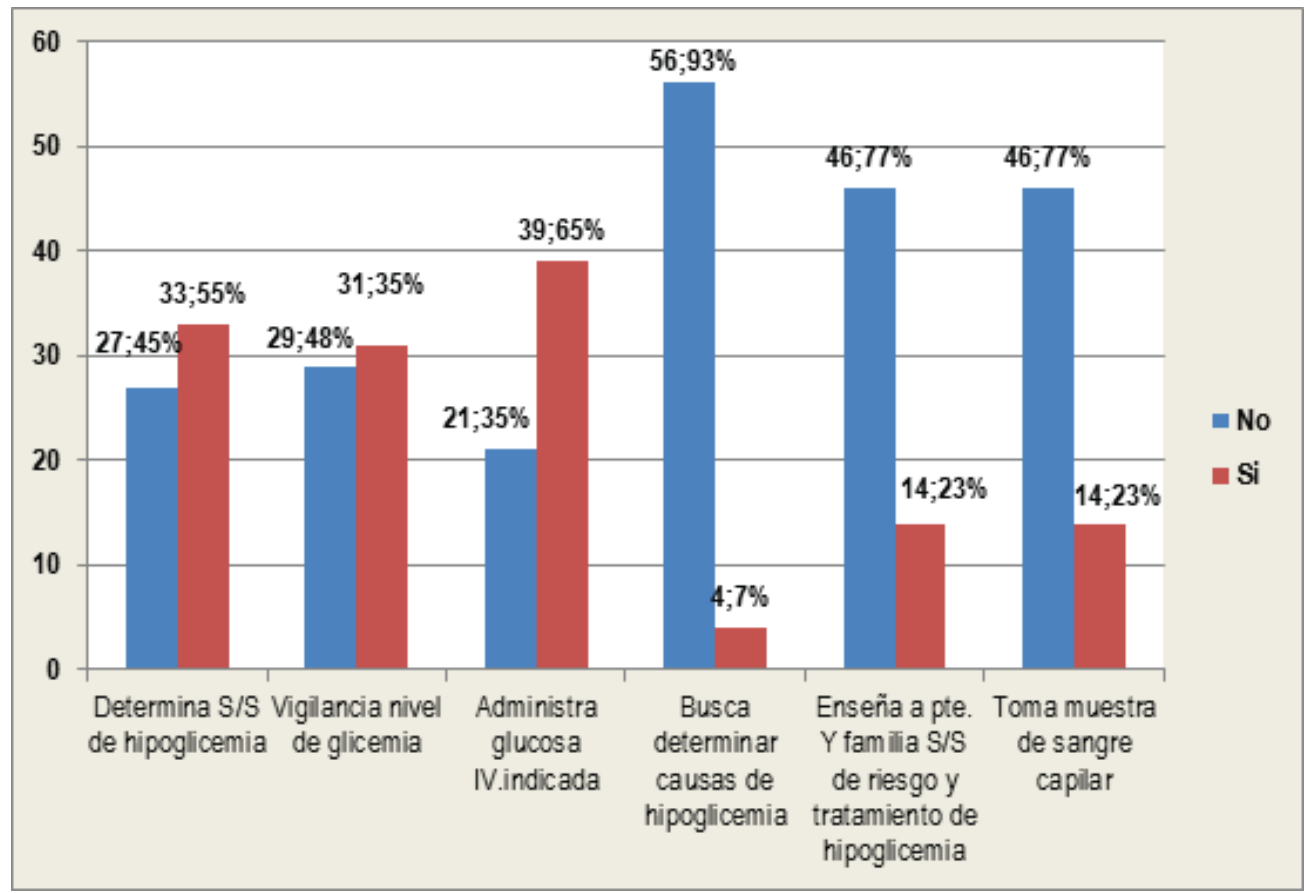

Fuente: Encuestas estudio de Variabilidad y calidad de la práctica de enfermería en pacientes con Diabetes mellitus en salas de Medicina Interna, HEU. 
Tabla 2. Intervenciones educativas de Enfermería a pacientes y familiares

\begin{tabular}{|l|l|c|c|}
\hline Descripción & Características & $\mathbf{N}^{\mathrm{a}}$ & $\%$ \\
\hline $\begin{array}{l}\text { Enseñanza alimentos } \\
\text { permitidos }\end{array}$ & $\begin{array}{l}\text { Instruye al paciente sobre alimentos } \\
\text { permitidos y no permitidos }\end{array}$ & 46 & 77 \\
\hline $\begin{array}{l}\text { Enseñanza cuidado de } \\
\text { los pies }\end{array}$ & Enseña técnica para arreglo de uñas & 40 & 67 \\
\hline Medicamento & Instruye uso de medicamento & 37 & 62 \\
\hline $\begin{array}{l}\text { Enseñanza de la dieta } \\
\text { prescrita }\end{array}$ & $\begin{array}{l}\text { Explica el propósito de llevar la dieta } \\
\text { prescrita }\end{array}$ & 26 & 43 \\
\hline $\begin{array}{l}\text { Enseñanza a valorar } \\
\text { color, temperatura y } \\
\text { humedad de la piel }\end{array}$ & $\begin{array}{l}\text { Enseña a valorar color, temperatura } \\
\text { y humedad de la piel }\end{array}$ & 24 & 40 \\
\hline Control de citas medicas & $\begin{array}{l}\text { Instruye sobre asistencia a control de } \\
\text { citas medicas }\end{array}$ & 24 & 40 \\
\hline $\begin{array}{l}\text { Extracción de muestra } \\
\text { de sangre }\end{array}$ & $\begin{array}{l}\text { Instruye extracción de muestra } \\
\text { capilar de sangre }\end{array}$ & 23 & 38 \\
\hline $\begin{array}{l}\text { Orientación a pacientes y } \\
\text { familiares }\end{array}$ & $\begin{array}{l}\text { Orienta a paciente y familiares sobre } \\
\text { proceso de la enfermedad }\end{array}$ & 22 & 37 \\
\hline $\begin{array}{l}\text { Orientación sobre } \\
\text { efectos del fármaco } \\
\text { utilizado }\end{array}$ & $\begin{array}{l}\text { Orienta sobre efectos del fármaco } \\
\text { utilizado }\end{array}$ & 18 & 30 \\
\hline Auto cuidado & $\begin{array}{l}\text { Enseña sobre autoanálisis y auto } \\
\text { cuidado }\end{array}$ & 17 & 28 \\
\hline Actividad física & $\begin{array}{l}\text { Enseña realización de actividad } \\
\text { física }\end{array}$ & 13 & 22 \\
\hline $\begin{array}{l}\text { Inclusión de la familia en } \\
\text { la educación }\end{array}$ & $\begin{array}{l}\text { Instruye a la familia del paciente a } \\
\text { participar del autocuidado }\end{array}$ & 11 & 18 \\
\hline
\end{tabular}

Fuente: Encuestas estudio de Variabilidad y calidad de la práctica de enfermería en pacientes con Diabetes mellitus en salas de Medicina Interna, HEU.

Educación sobre autocuidado y control de la diabetes: es uno de los aspectos más relevantes en el tratamiento integral de las personas que padecen esta enfermedad, el objetivo es lograr que el paciente sea capaz de mantener bajo control sus niveles de glucosa y pueda prevenir las complicaciones que la diabetes puede provocar a corto y a largo plazo además de permitir elevar su calidad de vida. 
Sin embargo, algunos de los pacientes admiten que nunca han recibido educación en salud por parte de enfermería, que este personal solamente se circunscribe en brindarle los medicamentos y que alguna de la información recibida es porque ellos se la solicitaron. Enfermería a si mismo señala que muy poca información le proporcionan a los familiares de estos pacientes y que influye en esta condición los horarios de visita ya que en ese momento casi no se les puede atender por la carga laboral. Rutina de cuidados que enfermería: según la descripción de las personas entrevistadas ellos realizan: Apoyo al paciente en su higiene personal la aplicación de medicamentos, aplicación de insulina medición de niveles de glucosa en sangre, curación de lesiones de piel y herida postquirúrgica en miembro amputado y orientación a pacientes y familiares sobre autocuidado abordando los temas acerca de ingesta de dieta alimenticia adecuada, control de peso, manejo de medicamentos y cuidado de los pies.

El estado del paciente se valora mediante la medición del nivel de glicemia en un $61,6 \%(37)$, revisan los signos vitales el 38\%(23). La principal intervención realizada para disminuir complicaciones en el paciente se focaliza principalmente en realizar la medición de la glicemia 68\%(41). Para asegurar que se brinde continuidad en los cuidados de Enfermería el personal realiza la entrega de turno $82 \%(49)$ y la formulación de la nota de enfermería 68\%(41).

La calidad en las actuaciones de enfermería se fundamenta en un modelo medico $43 \%$ (26) y en un modelo de enfermería $17 \%(10)$.

\section{Variabilidad enfermería en la atención al paciente con Diabetes mellitus.}

Variabilidad: Se define como aquellas diferencias en el proceso asistencial y/o en el resultado de la atención de un problema clínico concreto, entre diversos proveedores o uno mismo, una vez controlados los factores demográficos, socioculturales y de estado de salud" (Toro, 2005). La variabilidad en la práctica es un elemento que apoya la medición de la calidad, Se espera que exista cierta variabilidad en la práctica clínica que indica el ajuste a las características de la población del servicio 0 del sistema sanitario. El problema puede ser cuando la variabilidad sea en gran frecuencia.

El personal de enfermería participante del estudio identifica que en su práctica clínica en pacientes diabéticos existen condiciones tanto de índole de competencias profesionales en ellas como de carácter organizativo que provoca variaciones en la atención. 
Tabla 3. Intervenciones de Enfermería enfocadas a disminuir complicaciones en el paciente.

\begin{tabular}{|l|c|c|}
\hline Descripción & N & $\%$ \\
\hline Medición de glucemia & 41 & 68 \\
\hline Medición de temperatura corporal & 26 & 43 \\
\hline Medición de ingestas & 20 & 33 \\
\hline $\begin{array}{l}\text { Mantenimiento de dispositivos para acceso venoso. (Vía } \\
\text { permeable y limpia) }\end{array}$ & 9 & 15 \\
\hline Medición de excretas & 9 & 15 \\
\hline $\begin{array}{l}\text { Vigila los puntos de inserción de dispositivos en busca de } \\
\text { datos de inflamación, flebitis o infiltración durante su } \\
\text { permanencia. }\end{array}$ & 8 & 13 \\
\hline Realiza técnica aséptica al manipular el catéter venoso & 3 & 5 \\
\hline Medición de presión arterial & 3 & 5 \\
\hline Medición de pulso & 1 & 2 \\
\hline Medición de la respiración & 0 & 0 \\
\hline $\begin{array}{l}\text { Sigue los criterios establecidos ante cualquier procedimiento } \\
\text { invasivo incluidos el manejo de la vía aérea y la inserción de } \\
\text { sonda vesical. }\end{array}$ & 0 & 0 \\
\hline
\end{tabular}

Fuente: Encuestas estudio de Variabilidad y calidad de la práctica de enfermería en pacientes con Diabetes mellitus en salas de Medicina Interna, HEU. 


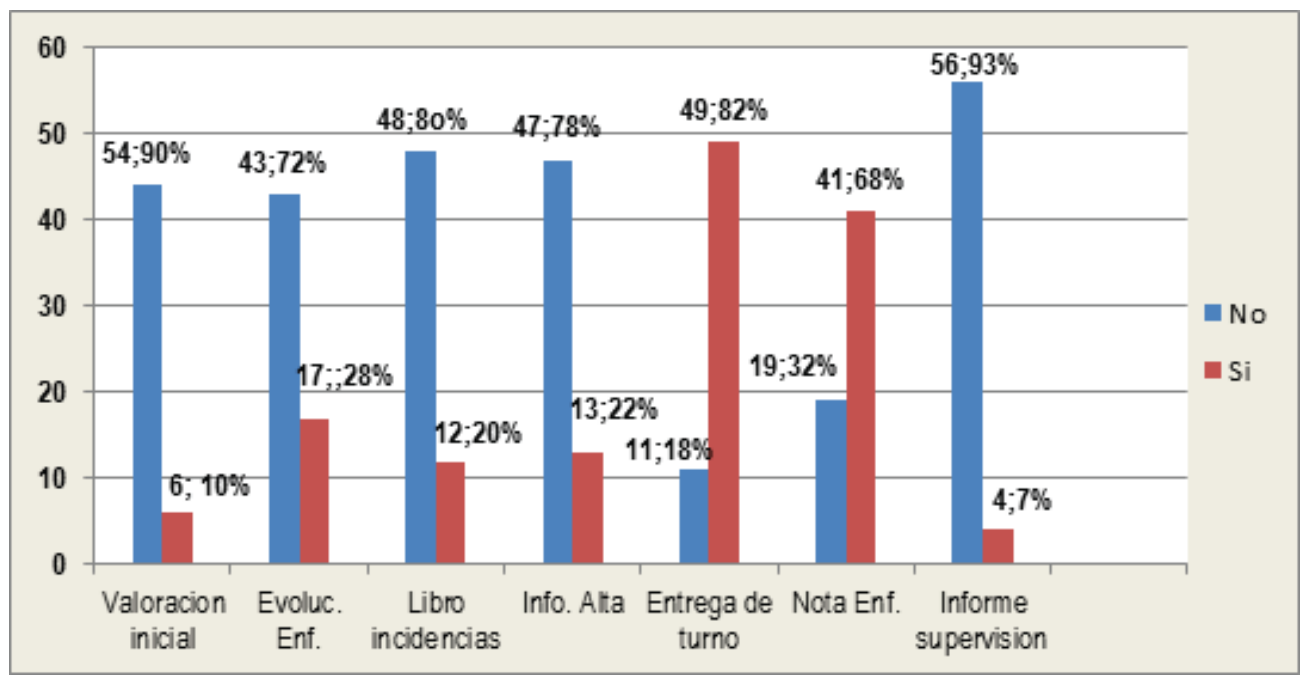

Fuente: Encuestas estudio de Variabilidad y calidad de la práctica de enfermería en pacientes con Diabetes mellitus en salas de Medicina Interna, HEU.

\section{Grafica 5. Fundamentos de la calidad asistencial de enfermería}

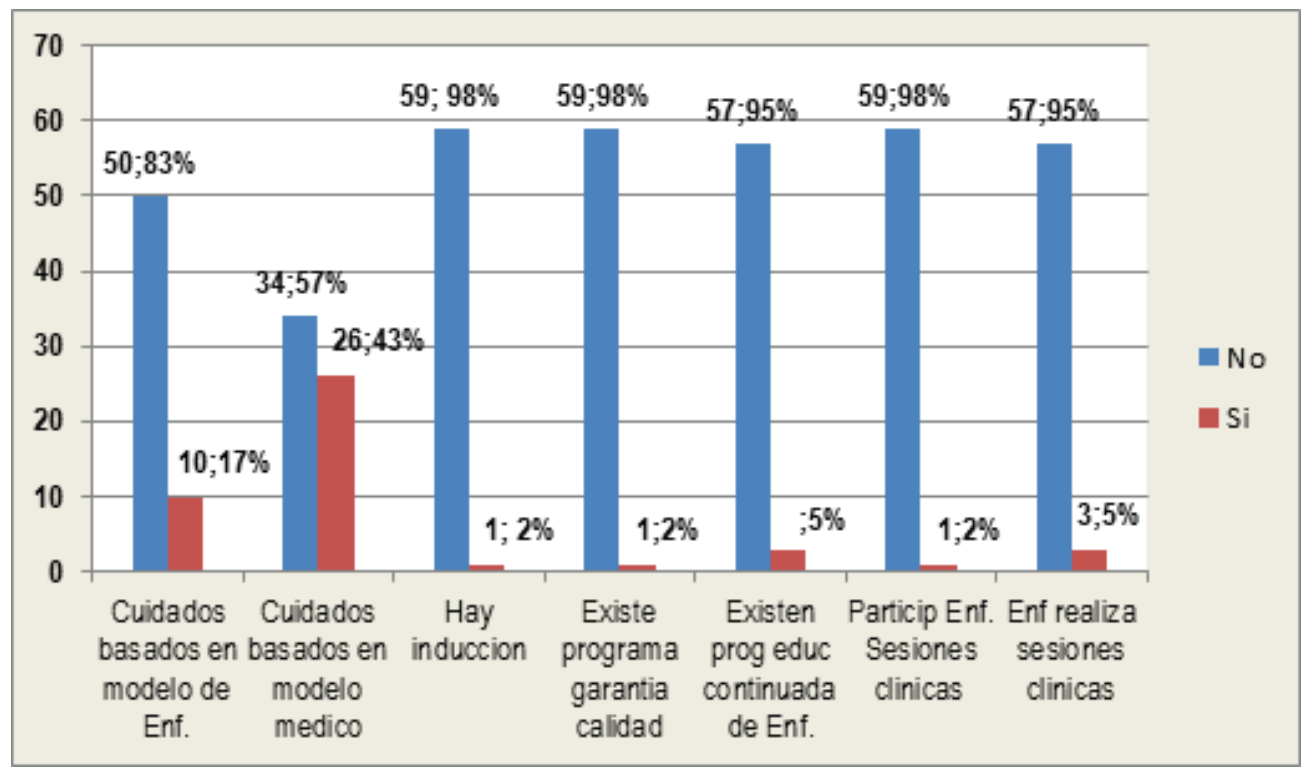

Fuente: Encuestas estudio de Variabilidad y calidad de la práctica de enfermería en pacientes con Diabetes mellitus en salas de Medicina Interna, HEU. 
En el estudio se identificó variabilidad en la práctica clínica de Enfermería al brindar las siguientes intervenciones:

1. En la valoración de signos y síntomas en el paciente con hiperglicemia o hipoglicemia. Para determinar si el paciente tiene hiperglicemia o hipoglicemia, una de las entrevistadas refiere que utiliza la entrevista y la medición de signos y síntomas, sin embargo la mayoría del personal admitieron que para valorar al paciente solo realizan la medición de la glicemia. El personal auxiliar de enfermería admite que en la valoración de los signos y síntomas para identificar híper o hipo glicemia tienen dificultad y consideran que son los mismos y que en tal sentido tienen complicaciones para determinar las intervenciones a realizar.

2. En los horarios para medición de glicemia por enfermería. En algunas salas los horarios de medición de glicemia varían según el estado del paciente, miden cada hora y hasta cada cuatro horas durante el turno de 8 horas.

3. En las técnicas para realización de curaciones en lesiones derivadas a neuropatías diabéticas. De lunes a viernes las curaciones son realizadas por licenciadas en enfermería, algunos fines de semana por estudiantes de la Carrera de Medicina en internado rotatorio o personal auxiliar de enfermería, no existe pauta que estandarice la ejecución de este procedimiento.

4. En la administración del medicamento insulina. La insulina es administrada conforme a la experiencia que cada uno como personal de enfermería posea y a las indicaciones que reciben por parte del personal médico las cuales son establecidas en el expediente clínico.

5. En contenido de la información que es brindada al pacientes con DM. y sus familiares durante se realiza la educación en salud.

Causas de la variabilidad: según personal de enfermería, las causas que explican la presencia de variabilidad en los cuidados de enfermería a pacientes con diabetes mellitus son: 1. Carencia en la formación para brindar los cuidados a pacientes con diabetes mellitus: los conocimientos que se tienen no son lo suficientes para brindar cuidados con evidencia, específicamente para el abordaje de educación en salud para el autocuidado de los pacientes, curaciones, aplicación de insulina.2. Estilo de práctica profesional: cada profesional sigue pautas personales basándose en su propia experiencia.3. Discontinuidad de cuidados: falta de continuidad de cuidados y comunicación entre los distintos niveles asistenciales e incluso entre los propios 
profesionales de una unidad (curaciones).4. Problemas de organización en el trabajo: poca implicación de las direcciones, ausencia de protocolos, normas etc.5.

\section{Déficit de recursos humanos e insumos para realizar los cuidados}

El $100 \%$ de este personal admitió no haber recibido educación continua sobre cuidados de enfermería a pacientes diabéticos, la información que apoya sus actuaciones son la información que les proporciona el personal médico, el internet y algunas veces se dejan llevar por "su instinto".

Cabe mencionar en estas unidades cada profesional sigue pautas personales basándose en su propia experiencia. Otro factor que según este personal incide es el déficit de recursos humanos e insumos para realizar los cuidados. Es relevante identificar el nivel de la incertidumbre entendida esta como la ausencia de evidencias científicas y/o consenso profesional sobre qué debe hacerse ante una situación concreta.

\section{Grafica 6. Elementos que condicionan variabilidad al brindar atención de Enfer- mería}

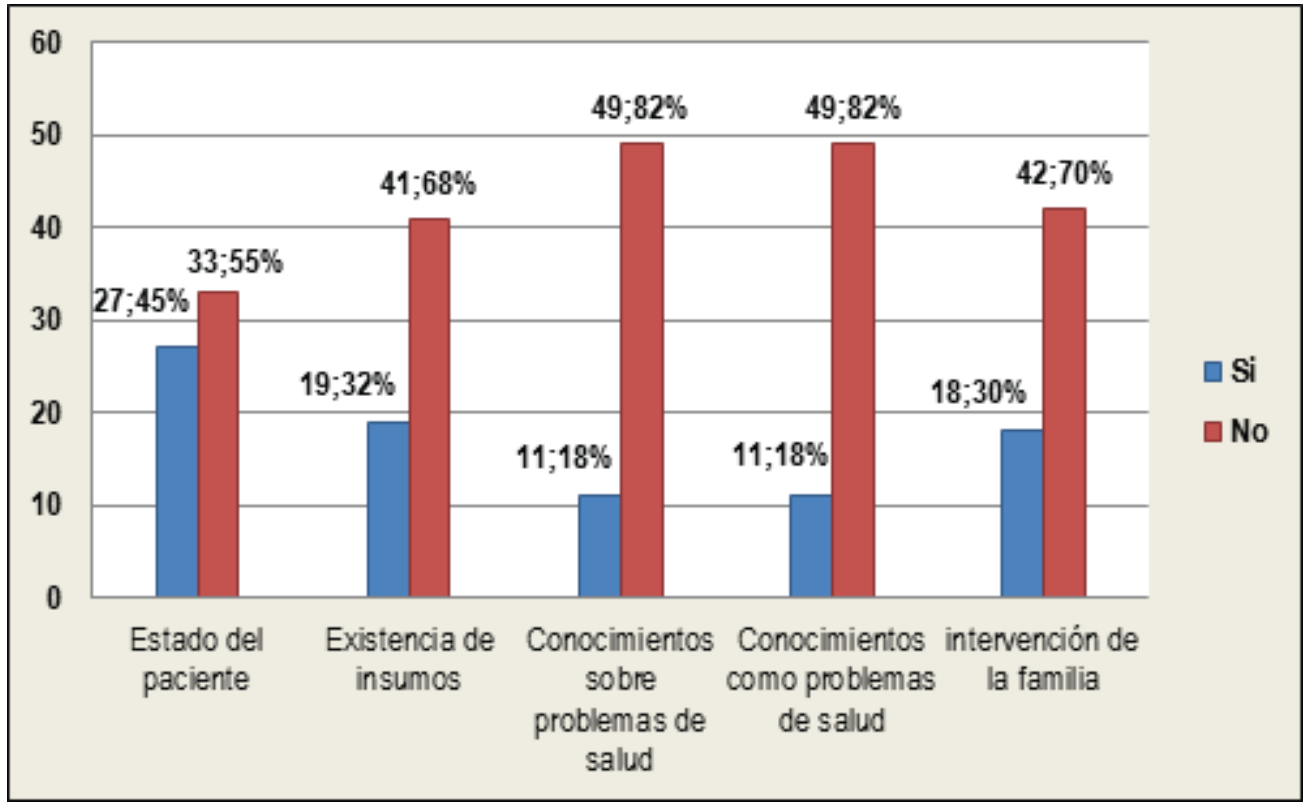

Fuente: Encuestas estudio de Variabilidad y calidad de la práctica de enfermería en pacientes con Diabetes mellitus en salas de Medicina Interna, HEU. 


\section{Nivel de satisfacción sobre la calidad de atención de enfermería en pacientes DM}

Seis de los diez pacientes entrevistados del estudio manifestaron estar satisfechos de la atención recibida por parte del personal de Enfermería en las Salas de Medicinas del HEU. "No pues con eso que este hay pendientes midiéndole cuanto tiene la azúcar que lo estén inyectando yo pienso que es una atención, yo le doy un 8".

Factores que provocan satisfacción a los pacientes acerca de los cuidados que reciben de Enfermería son: Una comunicación clara y efectiva, recibir información oportunamente, la competencia profesional un correcto de los medicamentos y recibir trato cortes y respetuoso.

Los factores que provocan insatisfacción: son de orden organizativo institucional como: la falta de insumos y medicamentos, por ser de suma necesidad ellos mismos deben buscar la forma de suministrárselos, dicha situación afecta su condición de salud y merma su economía. La carencia de insumos afecta también el estado en la comodidad del paciente en el caso del presente estudio afecta la condición de descanso de los pacientes "sí que estén pendientes de la ropita, a veces traigo sabanas de mi casa, se me mojan por mi condición, de repente a veces me hago pipi y se moja toda mi ropita y yo estoy muy mojada y estoy incomoda entonces es una de las incomodidades".

\section{Grafica 7. Percepción competencia profesional del personal de enfermería}

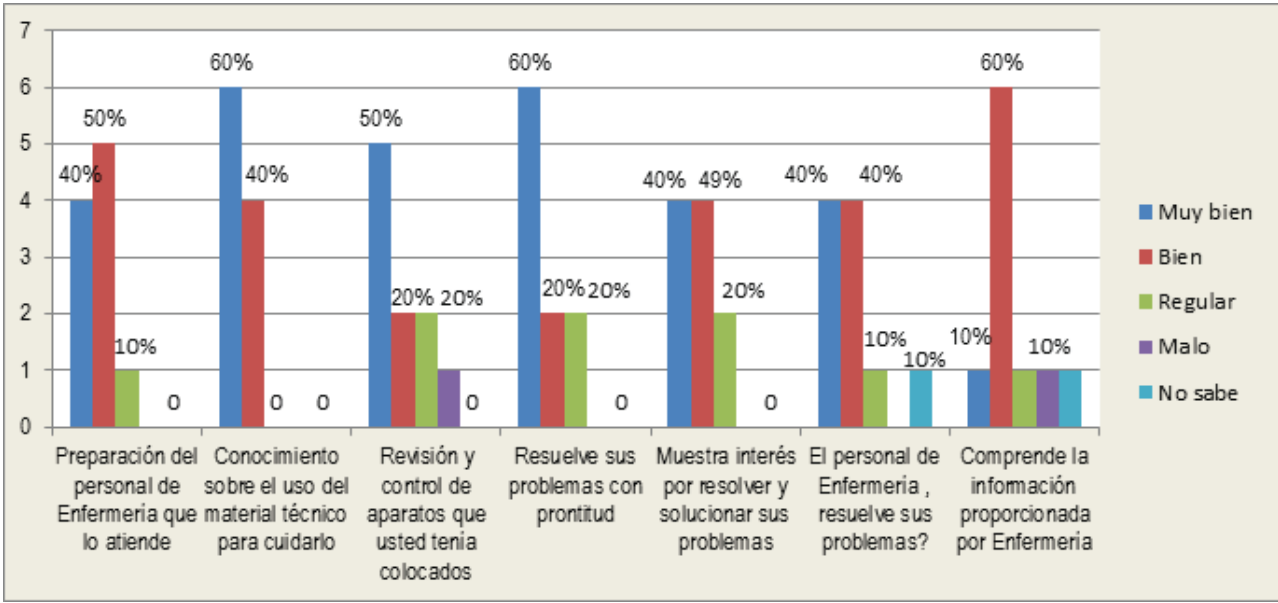

Fuente: Encuestas estudio de Variabilidad y calidad de la práctica de enfermería en pacientes con Diabetes mellitus en salas de Medicina Interna, HEU. 
Grafica 8. Comunicación: trato, servicio, empatía, seguridad, información, competencia y cortesía

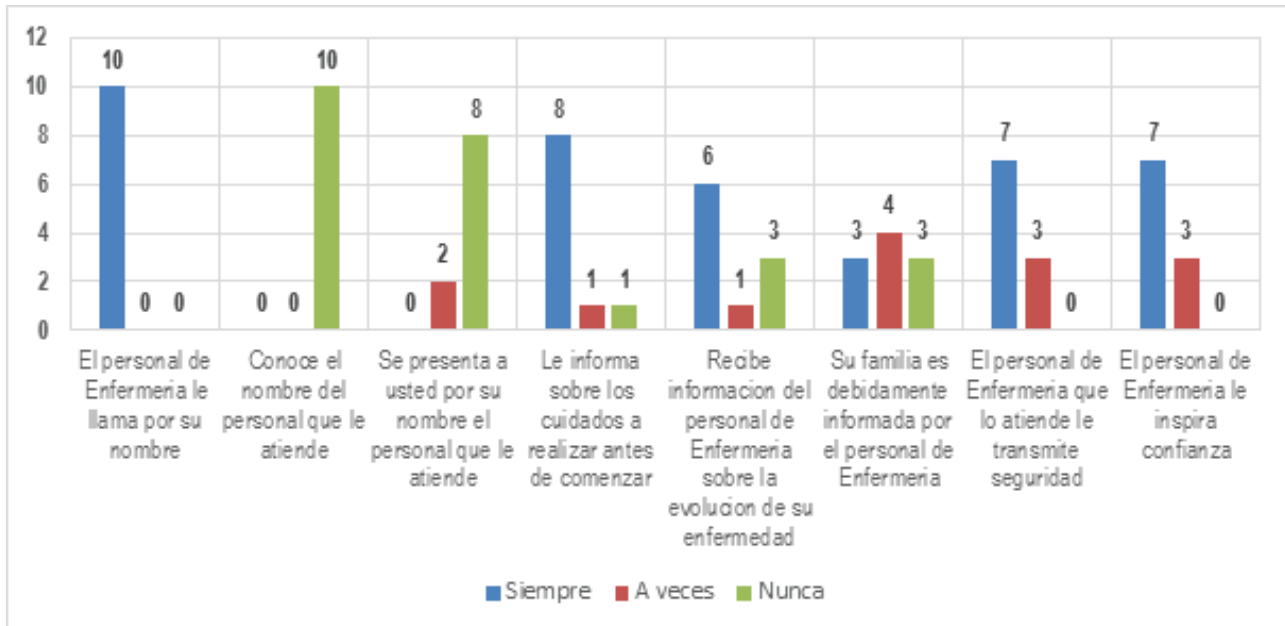

Fuente: Encuestas estudio de Variabilidad y calidad de la práctica de enfermería en pacientes con Diabetes mellitus en salas de Medicina Interna, HEU.

\section{Grafica 9. Comodidad: disponibilidad, confort, limpieza, empatía, tangibilidad}

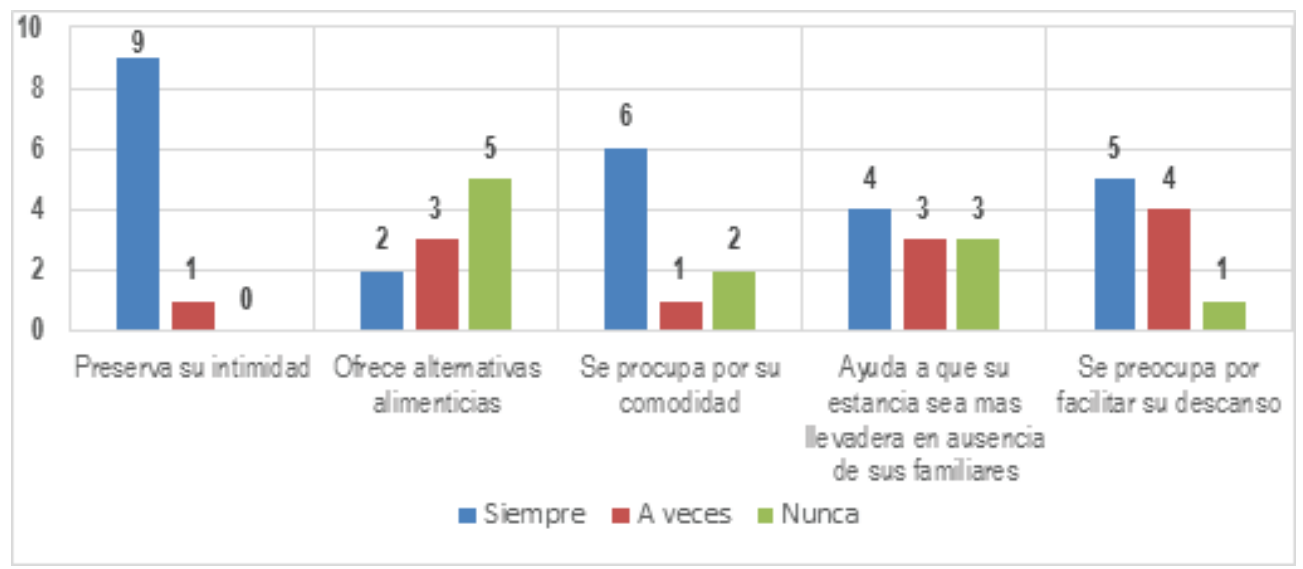

Fuente: Encuestas estudio de Variabilidad y calidad de la práctica de enfermería en pacientes con Diabetes mellitus en salas de Medicina Interna, HEU. 


\section{Fiabilidad en los cuidados de enfermería:}

Fiabilidad: es la capacidad que debe tener el profesional de enfermería en brindar un servicio de manera confiable, segura y cuidadosa.

Existe un nivel de satisfacción y confianza por parte de los pacientes hacia el grado de responsabilidad manifiesta por enfermería en sus acciones. "le voy a decir algo que las de enfermería parecen que fueran las mamas de nosotros, ahí están desvelándose para allá y para acá, y que más vamos hacer agradecerles por todo" cuidados no fiables: claro aquí viene bastante personal estudiante y necesitan bastante apoyo de uno.

Además, los pacientes opinan que el servicio de Enfermería tiene sobredemanda, por lo que piensan que el tiempo de espera para recibir atención de enfermería es más larga de lo establecido y al final ellos mismos tratan de resolver sus necesidades. "Para mi esta bueno pero como le digo aunque sea renqueando pero no trato de comprometerlas más yo a ellas, me voy a hacer mis necesidades allá al baño". "De repente hay demasiados pacientes uno no va a querer que solo con uno va estar el enfermero entiende tiene que atender los otros tal vez uno dice ya ratos me puso ese suero y se le olvido."

La comunicación clara y efectiva es esencial para transmitir confianza a los pacientes en especial cuando se trata de brindar información referente al estado de salud "Cada vez que me van a inyectar algo le pregunto que es para que es verdad, entonces por ejemplo me pusieron un medicamento y me dieron ganas de vomitar, y las enfermera me explico que así es" y en este sentido hay insatisfacción manifiesta.

El nivel de valoración sobre el estado de salud del paciente y la medicación son dos elementos de los cuidados de Enfermería que los pacientes certifican con importancia para el reconocimiento de cuidados de confianza y fiables. "Pues si porque a mí a media noche me ponen medicina las enfermeras me toman la diabetes, me pinchan el dedito si 4 veces al día porque cuando vine la tenía mal".

\section{Relaciones interpersonales enfermería y pacientes}

El estudio pone de manifiesto la existencia de buenas relaciones interpersonales entre Enfermería y algunos de los pacientes entrevistados "Las muchachas son muy atentas aquí", "también las enfermeras que quedan de turno son muy atentas si porque a mí en la noche no me viene a ver nadie y yo les pido ayuda, y ellas me 
ayudan", se refleja a través estas acciones el fomento de una relación interpersonal saludable con los pacientes, y se expresan a través de la comprensión incorporando además los elementos de comunicación y escucha efectiva.

Sin embargo no todos los pacientes experimentan el mismo nivel de satisfacción al respecto, existe insatisfacción debido a que algunos miembros del equipo de Enfermería presentan deficiencias para comunicarse de forma más eficaz con ellos : "mire hay unas que son pacientes, yo conocí una que ella no era paciente no me acuerdo en que sala esta, pero si será que no es completa, pero si se enoja si dice 'no se cuidan esto miren como vienen hasta que ya les van a cortar los pies', algunas que no pero esa como le digo que pareciera que no fuera completa" .La sobre carga emocional de Enfermería se manifiesta algunas veces en el trato que es percibido por los pacientes como arrogante "No pues como no todas las personas somos iguales hay personas que son más amables, otras que son más arrogantes".

\section{ANÁLISIS}

Existen variaciones en la atención de enfermería a pacientes con Diabetes mellitus específicamente en las intervenciones de valoración en los signos y síntomas, esta acción es esencial para que este personal pueda determinar el estado de salud del paciente con D.M., también hay variabilidad en los horarios de aplicación de medicamentos tales como la insulina y en las formas de proceder durante la ejecución de las curaciones esto se debe fundamentalmente a que en la sala no existen normas, guías clínicas para manejo de paciente con DM., así como lineamientos que estandaricen y evalúen los cuidados y la realización de procedimientos, esta condición de incertidumbre y poca evidencia en la toma de decisión limita la calidad de la atención en pacientes diabéticos por parte del personal de enfermería en las salas de Medicina Interna del Hospital Escuela Universitario.

Para incidir positivamente en la salud de los pacientes diabéticos se deben mejorar las competencias profesionales de enfermería en el manejo de estos mismos ya que a pesar que es un personal en su mayoría con más de 15 años de laborar en el servicio de Medicina Interna no han recibido educación continua en cuidados a paciente diabético.

El carecer de un modelo de enfermería, de programas para gestión de la calidad, de inducción para nuevo recurso humano, de la realización de sesiones clínicas de 
enfermería como base para la gestión clínica de los cuidados provoca que este personal adopte como primer criterio de sus actuaciones el modelo médico, deslegitimando el perfil profesional con el cual fue formado y de esta manera el usuario, y la institución de salud tienen perdida pues no se da una entrega de intervenciones de enfermería como un proceso planificado, continuo, evaluado y de cambio ,fundamentado en la evidencia científica que se constituye en la herramienta para obtener resultados de calidad.

Algunos de los factores que les provocan satisfacción de los cuidados que reciben de enfermería a los pacientes son los siguientes: comunicación clara y efectiva, recibir información oportuna, que el personal de enfermería muestre tener competencia profesional, manejo correcto de los medicamentos y que les brinde un trato cortes y respetuoso.

Los factores que les provocan insatisfacción son: son de orden organizativo institucional como la falta de insumos y medicamentos, que por ser de suma necesidad ellos mismos deben buscar la forma de suministrárselos, dicha situación afecta su condición de salud y refieren que merma su economía familiar.

Seis de los diez pacientes entrevistados refirieron estar satisfechos con la calidad de atención recibida del personal de enfermería.

\section{CONCLUSIONES}

1. Las intervenciones de enfermería que se realiza con mayor frecuencia es la medición de nivel de glucosa en sangre, a el nivel educativo la intervención más frecuente es la instrucción a pacientes y familiares en cuanto a la dieta alimenticia adecuada y el cuidado de los pies.

2. Existe variabilidad en la atención que brinda enfermería a pacientes con Diabetes mellitus y el personal de enfermería percibe estas variaciones las cuales argumentan se debe a la falta de educación continua.

3. La gestión clínica de enfermería en las salas estudiadas se fundamentan en un modelo médico y no de enfermería propiamente dicho lo cual impide que este personal desarrolle los cuidados bajo un modelo de atención conforme a su perfil profesional, además en estas salas no se desarrollan programas para la 
gestión de la calidad, inducción a personal, sesiones clínicas o se les a capacitado en los cuidados ha pacientes diabéticos, no existen guías clínicas que sirvan como pauta para estandarizar la atención, situación que incide negativamente en la calidad de los cuidados que este personal brinda.

4. El $100 \%$ del personal entrevistado no habían recibido formación específica sobre cuidados de enfermería a pacientes con Diabetes mellitus.

5. La mayoría de los pacientes con Diabetes mellitus interno en las salas de Medicina Interna del HEU., están satisfechos por la atención recibida de parte de Enfermería, los pacientes tienen una percepción positiva sobre el cuidado de enfermería.

\section{RECOMENDACIONES}

1. Formular estándares de atención en la práctica de enfermería así como evaluaciones de la calidad asistencial como herramientas para disminuir la variabilidad en sus actuaciones en los diferentes procesos asistenciales. Esta estandarización de los procesos se debe realizar mediante guías clínicas, protocolos, procedimientos. Esta condición a su vez facilita la medición de desempeño del profesional de la salud, promueve la auditoria de casos mediante indicadores del proceso.

2. Desarrollar para el personal de enfermería educación continua referente al desarrollo de los cuidados basados en evidencia con el propósito de proporcionar cuidados de calidad y conservar al máximo la seguridad del paciente.

\section{BIBLIOGRAFÍA}

Cañarejo Andrango Andrea Estefanía, P. (2013). Calidad de atencion que presta el personal de Enfermeria del sub centro de salud San Pablo. Tesis, Universidad Tecnica del Norte, Facultad Ciencias de la salud, Enfermeria, Ibarra.

Heredia, F. A. (2015). Calidad y auditoria en salud. En F. A. Heredia, Calidad y auditoria en salud. Bogota, Colombia. 
Hueso, B. H. (2015). Variabilidad en el manejo diagnostico y terapeutico de pacientes ingresados por un primer episodio de insuficiencia cardiaca, estudio multicentrico PREDICE. Sevilla. Recuperado de https://idus.us.es/xmlui/handle/11441/34618 Jesus Lopez Alcalde, X. B. (2008). Sobre la salud publica basada en pruebas. Revista Española de Salud Publica. Recuperado de http://www.redalyc.org/revista.oa?i$\mathrm{d}=170$

J.A. Esperón Güimil et al. (s.f.). Variabilidad en el abordaje de las heridas crónicas: ¿qué opinan las enfermeras? Recuperado de http://scielo.isciii.es/scielo.php?script=sci_arttext\&pid=S1134-928X2014000400007

Juan Francisco Menárguez Puche, J. A. (s.f.). Lectura crítica de una Guía de practica clinica. Murcia, España.

L. Quecedo, J. I. (2001). Herramientas de gestión clínica (I):Guias de practicas clinicas y vias clinicas. Actualizaciones de anestesiologia y reanimacion. Recuperado de www.fgcasal.org/publicaciones/LQI.pdf

M. Losada Otalora, A. R. (2007). Calidad del servicio de salud: una revision a la literatura desde la perspectiva del marketing. Recuperado de www.scielo.org.co/scielo.php?script=sci_abstract\&pid...35922007000200011

Reyes Luna, P. J. (2007). Adherencia de las enfermeras/o a utilizar un modelo teoricocomo base de la valoracion de enfermeria. Recuperado de www.scielo.cl/scielo.php?script=sci...pid...95532007000100006

Salud, O. M. (2014). Organizacion Mundial de la Salud, Nota descriptiva N³12. Recuperado de www.who.int/mediacentre/factsheets/fs312/es/

Toro, A. G. (2005). Variaciones en la Práctica Médica. Evidentia. Recuperado de https://www.toro.com/en/agriculture 